\title{
Reseña del libro: Epidemiología, diseño y análisis de estudios
}

\author{
Book review: Epidemiology, design and analysis of studies \\ Claudia T. Solano-Pérez ${ }^{a}$, Jesús C. Ruvalcaba-Ledezma ${ }^{b}$
}

\begin{abstract}
:
This review highlights the titled work. Epidemiology, design and analysis of studies. From a structural analysis, which denotes the importance of training in epidemiology from its usefulness in teaching at the bachelor's level and postgraduate degrees in Public Health and Biomedical and Health Sciences, as well as the invitation to staff teacher of subjects such as epidemiology, research methodology and statistics, since these as areas of knowledge cannot be unlinked.
\end{abstract}

\section{Keywords:}

Epidemiology, study analysis.

\section{Resumen:}

En esta reseña se resalta la obra titulada. Epidemiología, diseño y análisis de estudios. A partir de un análisis estructural, misma que denota la trascendencia de la formación en epidemiología a partir de su utilidad en la enseñanza a nivel de licenciatura y de los posgrados en Salud Pública y Ciencias Biomédicas y de la Salud, así como la invitación al personal docente de asignaturas como epidemiología, metodología de la investigación y de estadística, ya que estas como áreas del conocimiento no pueden estar desvinculadas.

\section{Palabras Clave:}

Epidemiología, análisis de estudios.

\section{Introducción}

La obra del reconocido autor, Mauricio Hernández Ávila, recopila elementos muy interesantes sobre diversas temáticas que conforman estructuralmente a la Salud Pública, entre ellas el autor compilador ha agregado elementos de metodología de la investigación, y estadística analítica, resulta contundente señalar, que estas dos áreas en conjunto constituyen los brazos de la epidemiología y que describimos a continuación.

\section{Presentación}

El libro Epidemiología, Diseño y análisis de estudios, es una obra que cuenta 385 páginas, en su edición de 2009 tuvo un tiraje de 1000 ejemplares, auspiciado por la Editorial Médica Panamericana. En su interior presenta el prólogo, la introducción y los contenidos temáticos divididos en 16 unidades.

Se inicia con un desarrollo conceptual e histórico de la epidemiología y pone de manifiesto el tema de los diseños de estudios epidemiológicos, donde el autor inserta una estructura conceptual de cada uno de los diseños; los estudios descriptivos transversales y sus variantes, los estudios de cohorte, los estudios de casos y controles, los ensayos aleatorizados, los estudios ecológicos, en cada

Autor de Correspondencia, Universidad Autónoma del Estado de Hidalgo, https://orcid.org/0000-0003-4648-981X, Email: claudia_solano@uaeh.edu.mx

b Universidad Autónoma del Estado de Hidalgo, https://orcid.org/0000-0002-5593-3946, Email: dcspjcarlos@gmail.com 
diseño hace hincapié mediante ejemplos prácticos y comprensibles.

Posteriormente, señala las principales medidas en epidemiología y estadística, como los son; tasas, razones, medidas de mortalidad, de morbilidad, de asociación, de diferencia, de impacto y/o de riesgo a partir de ejemplos o ejercicios sobre ello, posteriormente se dedica a explicar conceptualmente y de manera operativa los estudios clínicos experimentales, donde se desglosan sus características, la pertinencia y factibilidad de estos, así como el análisis de ellos.

Los capítulos V, VI, VII y VIII se refieren a tipos de diseños de investigación en una narrativa ampliada con sus características, clasificaciones y formas de medición: ensayos clínicos aleatorizados, estudios de cohorte, estudios de casos y controles, encuestas transversales. Posterior a ello, el tema que resalta es la Investigación de brotes. Y se cierra ese bloque con el capítulo de sesgos, describiendo los siguientes: de selección, de información, de confusión, y finalmente algunos ejercicios.

El último bloque que comprende los capítulos del XI al XVI, se refieren a temas relacionados con la estadística aplicada: Introducción al análisis estadístico, Regresión lineal simple, Regresión lineal múltiple, Regresión logística, Análisis de supervivencia, y, por último, Interacción o modificaciones de efecto en modelos de regresión lineal y logística.

\section{Recomendación para los lectores}

Por tratarse de una obra tan versátil y a la vez completa, esto podría dificultar su comprensión dependiendo del nivel educativo de quien se acerca al texto; supongamos que un estudiante de la Licenciatura de Médico Cirujano tuviera acceso a este libro; requeriría conocimientos básicos de epidemiología y estadística para comprender los primeros capítulos: plagas, epidemias, paradigma de la red causal y funciones fundamentales de la epidemiología moderna.

Estudiantes de la Maestría en Salud Pública alcanzarían a comprender conceptos a nivel intermedio de este texto, pero bajo la tutela del docente conocedor de los contenidos de este texto, lo mismo que los alumnos de la Maestría en Ciencias Biomédicas y de la Salud. Para estudiantes de Licenciatura de Médico Cirujano, al llevar la asignatura de bioestadística podrán comprender el capítulo III: principales medidas, pero con mayor dificultad lograrán entender el capítulo XIV con temática referente a Regresión logística.
Si bien el texto presenta de manera comprensible la información, para estudiantes de nivel licenciatura su utilidad es limitada, ya que en los estudiantes de nivel superior se observa la falta de cursos básicos de estadística donde sean del dominio de estos y la comprensión respecto a la estadística y su interpretación se requiere de instrumentos verbales como antecedente. En nivel posgrado este libro representa un área de oportunidad para incrementar precisamente elementos verbales.

Por lo anterior es que el egresado de posgrado emprende el camino hacia la investigación, proceso que requiere el dominio de los diseños de estudio y el análisis estadístico en concordancia con el diseño de su investigación, es entonces que se hace necesario que el profesor de metodología de la investigación domine la parte estadística y que este domine de igual forma la metodología de la investigación, por lo que se les invita a los docentes tanto de nivel licenciatura como de nivel de maestría a consultar este libro de texto para enriquecer su lenguaje y construir este con sus estudiantes de manera más efectiva

\section{Conclusión}

La epidemiología fundamenta su operatividad a partir de la interrelación e interacción entre áreas académicas como lo son la metodología de la investigación y la estadística, dichas áreas las expone de manifiesto el autor y esto le confiere a la obra presentada aquí en el formato de reseña un valor de uso en el que el docente de epidemiología-salud pública requiere coincidir en el proceso de la enseñanza a partir de la estructura que el autor denota en su valiosa obra y que evitaría confusión en el aprendizaje y la operatividad de la epidemiología por parte de los estudiantes.

\section{Referencia}

[1] Hernández-Ávila Mauricio. Epidemiología, diseño y análisis de estudios. 385 pp. Editorial Médica Panamericana. 2009. 